\title{
Eine Typologie für die vergleichende Wahlsystemforschung*
}

\section{Kurzfassung}

Die Vergleichende Politikwissenschaft wartet mit einer Reihe von Typologie-Ansätzen für Wahlsysteme auf, welche sich jeweils entweder auf das Repräsentationsprinzip als Gesamtziel eines Wahlsystems oder auf dessen technische Ausgestaltung konzentrieren. Dieses Nebeneinander verschiedener Ansätze sorgt für Konfusion bei der Einordnung von Wahlsystemen und deren Vergleich, da sich unterschiedliche Studien auf verschiedene Basen stützen und somit nur sehr eingeschränkt vergleichbar sind. Dieser Beitrag begegnet dieser Problematik, indem eine allgemeine Typologie entworfen wird, welche sowohl das Repräsentationsprinzip als auch die technische Dimension von Wahlsystemen bei deren Einordnung berücksichtigt. Dabei ist die Einsicht leitend, dass aufgrund der Komplexität der Wirkungen von Wahlsystemen ein bestimmtes generelles Ziel nicht die technische Ausgestaltung determiniert und umgekehrt. So wird der vergleichenden Wahlsystemforschung ein vollständiger typologischer Rahmen geliefert. Basierend auf dieser mehrdimensionalen Typologie gelingt es anhand der Einordnung konkreter Fallbeispiele Folgendes zu zeigen: 1) Jegliche Wahlsysteme lassen sich mit Hilfe der Typologie einordnen und vergleichen; 2) bei Berücksichtigung des Repräsentationsprinzips ist es möglich, den Erfolg von Wahlsystemen unabhängig von einer normativen Grundhaltung zu bewerten; 3) bei der Analyse eines Wahlsystems muss jeweils sowohl dessen Repräsentationsprinzip als auch dessen technische Ausgestaltung berücksichtigt werden, um Fehlschlüsse zu vermeiden.

* Dieser Beitrag entstand im Rahmen des von der DFG geförderten Projektes „Der funktionale Vergleich von Wahlsystemen unter besonderer Berücksichtigung von Mischwahlsystemen“. Die Autoren danken der DFG für die finanzielle Förderung. Wir danken außerdem der Redaktion der Zeitschrift für Politikwissenschaft sowie den anonymen Gutachtern für hilfreiche Anmerkungen. 


\section{Inhalt}

1. Einleitung 494

2. Grundbegriffe der Wahlsystemforschung 496

3. Diskussion und Problematisierung bestehender Typologie-Ansätze 499

4. Anforderungen an eine allgemeine Typologie von Wahlsystemen 502

5. Entwurf einer Typologie bei gleichzeitiger Berücksichtigung von Repräsentationsprinzip und technischer Ausgestaltung 505

a) Konstruktion eines allgemeinen typologischen Rahmens 505

b) Berücksichtigung technischer Multidimensionalität 507

6. Einordnung konkreter Fallbeispiele $\quad 510$

7. Fazit 518

\section{Einleitung}

„Wahlen bilden die Grundlage des modernen Demokratieverständnisses“ (Nohlen 2009 a: 27) und „sind die zentrale Institution der repräsentativen Demokratie“ (Schmitt-Beck 2012: 4). Die Art und Weise, wie sich die Präferenzen der Wähler in Stimmen für verschiedene Parteien beziehungsweise Kandidaten ausdrücken, und wie aus diesen Stimmen die Zusammensetzung des Parlaments zu ermitteln ist, wird maßgeblich durch die Ausgestaltung des Wahlsystems beeinflusst (Gallagher/ Mitchell 2005 b: 3). Die Wahlsystemforschung sowie die Wahlforschung, die mit der Wahlsystemforschung eng verknüpft ist, sind mittlerweile reich an Beiträgen zu diversen Forschungsfragen, die sich genau mit diesen Zusammenhängen zwischen Präferenzen, Stimmen und Sitzverteilungen befassen. Zu nennen sind hier etwa Arbeiten mit Bezug auf strategische Anreize, die Wahlsysteme Wählern liefern (z. B. Cox 1997), auf die Untersuchung strategischen Wahlverhaltens (z. B. Gschwend 2007) oder auch, welche Strategien Parteien in Wahlkämpfen abhängig vom Wahlsystem entwickeln (z. B. Ferrara/Herron/Nishikawa 2005). Dieser Reichhaltigkeit an Forschungsbeiträgen steht jedoch ein Mangel eines gemeinsamen typologischen Rahmens gegenüber. Seit Jahrzehnten wird das Bedauern darüber geäußert, dass keine Einigkeit darüber besteht, wie verschiedene Wahlsysteme zu typologisieren sind. Insbesondere die Einordnung sogenannter Mischwahlsysteme sorgt immer wieder für Kontroversen (Kaiser 2002; Nohlen 2009 a). So ist gerade aus deutscher Sicht auffällig, dass die personalisierte Verhältniswahl von prominenter Seite als 
Eine Typologie für die vergleichende Wahlsystemforschung

Mischwahlsystem gesehen wird, ${ }^{1}$ von ebenso prominenter Seite als Verhältniswahlsystem. ${ }^{2}$

Nachfolgend versuchen wir, diese Forschungslücke zu füllen und den vielversprechenden einzelnen Forschungsbeiträgen einen solchen gemeinsamen Rahmen zu geben, indem wir eine allgemeine und vollständige Typologie von Wahlsystemen entwerfen. Diese synthetisiert die verschiedenen bisherigen Typologie-Vorschläge und bietet zudem die nötige Flexibilität, um an konkrete Forschungsfragen jeweils gewinnbringend angepasst werden zu können. Zum einen wird berücksichtigt, welches Repräsentationsziel einem Wahlsystem zugrunde liegt (vgl. Nohlen 1984, 2009 a für einen Typologie-Ansatz, welcher sich an den Effekten von Wahlsystemen und den damit erreichbaren Zielen orientiert). Zum anderen ist in Betracht zu ziehen, wie ein Wahlsystem technisch aufgebaut ist (vgl. z. B. Massicotte/Blais 1999; Shugart/Wattenberg 2001 a; Gallagher/Mitchell 2005 a für einen Typologie-Ansatz, welcher sich an den technischen Elementen von Wahlsystemen orientiert). Der Mehrwert des typologischen Rahmens ergibt sich aus 1) der nun gegebenen Inklusion aller theoretisch möglichen wie praktisch vorkommenden Wahlsysteme; 2) der Ableitung theoretischer Erklärungsansätze für die Ausgestaltung von Mischwahlsystemen; 3) der über den gemeinsamen (Bewertungs-)Rahmen erfolgenden Verbindung von Einzelfallstudien und komparativ-quantitativen Studien; 4) der Möglichkeit, über Erfolg und Misserfolg von Wahlsystemen urteilen zu können, ohne dabei auf normative Prinzipien rekurrieren zu müssen.

Basierend auf einer Klärung der Grundbegriffe der Wahlsystemtheorie (Abschnitt 2) diskutieren und problematisieren wir bestehende Typologie-Ansätze (Abschnitt 3). Aus dieser Diskussion leiten wir sowohl inhaltliche als auch methodologische Anforderungen an eine allgemeine Typologie von Wahlsystemen ab (Abschnitt 4) und entwickeln eine solche Typologie unter gleichzeitiger Berücksichtigung des Repräsentationsprinzips und der technischen Ausgestaltung (Abschnitt 5). In Abschnitt 6 nutzen wir eine konkrete Spezifizierung der allgemeinen Typologie und füllen den typologischen Rahmen, indem wir ausgewählte Fallbeispiele einordnen und die Notwendigkeit einer multidimensionalen Betrachtung unterstreichen.

1 Massicotte/Blais 1999; Kaiser 2002; Gallagher/Mitchell 2005 b; Linhart 2009; Pappi/Herrmann 2010.

2 Lijphart/Grofman 1984 a; Lijphart 1994; Sartori 1997; Norris 2000; Nohlen 2009 a; Behnke 2011; Decker 2011. 


\section{Grundbegriffe der Wahlsystemforschung}

Zunächst ist festzustellen, dass die dichotome Gegenüberstellung von Mehrheitsund Verhältniswahl längst einer differenzierteren Betrachtung gewichen ist, welche die Existenz gradueller Unterschiede berücksichtigt und zu dem Schluss kommt, dass mit Mischwahlsystemen eine dritte Kategorie von Wahlsystemen in Analysen einzubeziehen ist (Shugart 2005). Die Unklarheit der Abgrenzung dieser Mischwahlsysteme von reinen Varianten der Mehrheits- und Verhältniswahl ${ }^{3}$ hat mitunter dazu geführt, dass ein wesentlich grundlegenderes Einordnungsproblem übersehen wurde. Nach welcher Regel lässt sich festlegen, welches Wahlsystem nun ein Mehrheits- oder ein Verhältniswahlsystem ist bzw. - im Falle von Mischwahlsystemen - einem dieser Typen zumindest näherkommt? Je nachdem, ob man sich vor allem an einer (erwarteten) Gesamtwirkung eines Wahlsystems oder an dessen technischer Gestaltung orientiert, kann man hier zu verschiedenen Einschätzungen kommen.

Der erstgenannte Ansatz blickt auf das Repräsentationsprinzip eines Wahlsystems. Hierbei geht es darum, ob ein Wahlsystem (zunächst unabhängig von seiner exakten technischen Ausgestaltung) primär das politische Ziel der Konzentration (also möglichst die Bildung von direkt durch die Verteilung der Wählerstimmen legitimierten Ein-Parteien-Regierungen) oder das der Proportionalität $t^{4}$ (also einer möglichst genauen Abbildung gesellschaftlicher Interessen im Parlament) verfolgt (Nohlen 2009 a: 140 ff.; Farrell 2011: 10 f.). ${ }^{5}$ Das Repräsentationsprinzip fungiert somit sowohl als Orientierung für die technische Gestaltung als auch als Bewertungsmaßstab der tatsächlichen Ergebnisse, welche durch das Wahlsystem hervorgerufen werden (Nohlen 1984: 88, 2009 a: 143).

3 Nohlen (2009 a: 130) etwa sieht Mischwahlsysteme als „Residualkategorie“ an; vgl. auch Behnke 2011.

4 Oft wird dieses Ziel mit dem Terminus „Repräsentationsfunktion“ benannt (etwa bei Nohlen 2009 a). Dies ist inhaltlich angemessener, da neben dem Ziel der Proportionalität auch weitere Vorstellungen wie etwa das der parlamentarischen Repräsentation von Minderheiten verfolgt werden. Da aus sprachlicher Sicht die Verwendung des Begriffs der Repräsentation als eines der Repräsentationsprinzipien eher verwirrend als hilfreich ist, verwenden wir im Folgenden dennoch den Begriff „Proportionalität“ für dieses Ziel.

5 Natürlich sind weitere Zielvorstellungen von Wahlsystemen vorstellbar, etwa eine Einflussnahme der Wähler auf die personelle Zusammensetzung des Parlaments (vgl. Nohlen 2009 a für die möglichen Funktionen von Wahlsystemen). Allerdings besteht die grundsätzliche Frage bei der Entscheidung für oder gegen ein Wahlsystem genau aus der Wahl, welches der beiden Repräsentationsprinzipien die zentrale Rolle einnehmen soll. Beide Prinzipien lassen sich nach dieser grundsätzlichen Entscheidung dann mit weiteren Zielen verknüpfen. Die überragende Bedeutung der beiden hier vorgestellten Repräsentationsprinzipien zeigt sich auch in der Expertenumfrage von Bowler/ Farrell/Pettitt (2005: 10 ff.). 
Eine Typologie für die vergleichende Wahlsystemforschung

Eine direkte Orientierung an der technischen Ausgestaltung eines Wahlsystems stellt weniger grundlegende Fragen, sondern zerlegt Wahlsysteme vielmehr in ihre (technischen) Einzelteile. Basierend auf Arbeiten von Rae (1967) und Nohlen (1978, 2009 a) lassen sich nach Schoen (2005) Wahlsysteme im Wesentlichen über deren Wahlkreisgröße, die Form der Kandidatur, die Stimmgebung und den Stimmverrechnungsmodus charakterisieren. Die Wahlkreisgröße beschreibt die Anzahl der zu wählenden Abgeordneten in dem Wahlkreis. Ein Wahlsystem kann hierbei durchaus auch Wahlkreise unterschiedlicher Größe beinhalten, die entweder nebeneinander existieren und/oder auf verschiedenen Ebenen miteinander verknüpft sein können (sogenannte tiers; Blais/Massicotte 1997). Im Falle mehrerer Ebenen ist die Form der Verknüpfung relevant. So ist ein Wahlsystem, welches Disproportionalitäten zwischen Stimmen- und Sitzanteilen der Parteien in kleinen Wahlkreisen mithilfe größerer Wahlkreise der oberen Ebene ausgleicht, ein kompensatorisches System. Die Kompensation kann hierbei nur anteilig oder auch voll erfolgen (Linhart 2009: 643). Ein Wahlsystem, welches keine solche Kompensation vornimmt, sondern bei dem die Stimmen auf den einzelnen Ebenen unabhängig voneinander verrechnet werden, bezeichnet man als paralleles Wahlsystem oder Grabenwahlsystem.

Bei der Form der Kandidatur sind die Einzel- und die Listenkandidatur zu unterscheiden, bei letzterer zusätzlich verschiedene Listenformen. Die Stimmgebung umfasst zum einen die Frage, wie viele Stimmen pro Wähler vorgesehen sind, zum anderen Details des Stimmgebungsverfahrens wie Möglichkeiten des Stimmensplittings, des Kumulierens, des Panaschierens oder der Übertragbarkeit von Stimmen. Der Verrechnungsmodus der Stimmen lässt sich allgemein unterteilen in Mehrheitswahl und Verhältniswahl (Schoen 2005), wobei diese Oberkategorien dann im Falle der Mehrheitswahl in die relative und absolute Mehrheitswahl und im Falle der Verhältniswahl je nach der verwendeten Methode (z. B. d'Hondtsches Höchstzahlverfahren, Hare-Niemeyer-Verfahren usw.) unterteilt werden können (Lijphart/Grofman 1984 b; Shugart/Wattenberg 2001 b; Gallagher/Mitchell 2005 b). Weitere Stellschrauben bilden Sperr- und Grundmandatsklauseln sowie die Möglichkeit, Wahlkreise insofern unterschiedlich zuzuschneiden, als die Zahl der durch einen Abgeordneten repräsentierten Bürger zwischen den Wahlkreisen variiert (sogenanntes malapportionment; ausführlicher Schoen 2005).

Folgt man Nohlens (2009 a: 140 ff.) Verständnis, nach dem die technischen Elemente ausschließlich Mittel zum Zweck der Erfüllung eines der Repräsentationsprinzipien darstellen, so lassen sich für die reinen Wahlsystemtypen Kongruenzen der Einteilung nach technischer Ausgestaltung und nach Repräsentationsprinzip feststellen. Das Prinzip der Proportionalität ist mit der reinen Verhältniswahl ver- 
bunden, in welcher genau ein landesweiter Wahlkreis existiert, in dem alle $n \mathrm{Ab}$ geordneten zu wählen sind (Wahlkreisgröße $n$ ), wobei $n$ der Parlamentsgröße entspricht. Die Zuteilungsverfahren der Verhältniswahl zielen dann genau darauf ab, die Verteilung der Wählerschaft proportional in den Sitzverhältnissen des Parlaments abzubilden. In der reinsten Variante der Mehrheitswahl hingegen existiert nicht ein Wahlkreis mit $n$ zu vergebenden Mandaten, sondern $n$ Wahlkreise mit je einem zu vergebenden Mandat. Hierbei sind Einzelkandidaturen vorgesehen; das Parlament setzt sich zusammen aus allen in den Wahlkreisen gewählten Kandidaten. Ist die Bedingung erfüllt, dass nationale Parteien (nicht viele regionale) jeweils in allen oder zumindest hinreichend vielen Wahlkreisen die aussichtsreichsten Kandidaten stellen, ist zu erwarten, dass ein solches Mehrheitswahlsystem das Repräsentationsprinzip der Konzentration bestmöglich erfüllt, indem es Mehrheiten für eine einzelne Partei generiert und damit Ein-Parteien-Regierungen hervorbringt (Duverger 1984; Kaiser 2002: 1556).

Eine solche ex ante-Verbindung von technischen Wahlsystemen und bestimmten Repräsentationsprinzipien ist jedoch auch bei technischer Eindeutigkeit nur sehr bedingt möglich, da Wahlsysteme in ihren Wirkungen stark von den jeweiligen sozio-politischen Rahmenbedingungen abhängen (Cox 1997: 13 ff.; Benoit 2002). Hierbei ist zwischen direkten (mechanischen) Wirkungen - also der rein mathematischen Umrechnung der Stimmen in Sitze - und indirekten (psychologischen) Wirkungen - dem Einfluss des Wahlsystems auf die Erwartungen und dementsprechenden strategischen Handlungsweisen von Wählern und Parteien - zu unterscheiden (Schoen 2005: $584 \mathrm{ff}$.). Letztere sind demnach auch dafür verantwortlich, dass die Wirkung eines Wahlsystems im Detail schwer zu prognostizieren ist. Je nach beispielsweise der geographischen Verteilung von politischen Präferenzen oder der Anzahl und Überlappung relevanter sozialer Konfliktlinien (Lipset/Rokkan 1967), können die gleichen technischen Regeln andere Anpassungsstrategien der relevanten Akteure hervorrufen (Cox 1997). So führt etwa ein relatives Mehrheitswahlsystem eben nur bedingt zu einer Konzentration des Parteiensystems im Sinne einer Ein-Parteien-Mehrheit, wenn in verschiedenen Wahlkreisen verschiedene (regionale) Parteien gegeneinander antreten (etwa im Fall Kanadas; Massicotte 2005). Gleichfalls kann eine reine Verhältniswahl dieses Ziel erfüllen, wenn die Interessen der Wählerschaft tatsächlich mithilfe zweier großer Parteien abgebildet werden können (hier ist Malta zu nennen, wo zwar keine reine Verhältniswahl vorliegt, allerdings in Mehrpersonenwahlkreisen nach Single Transferable Vote gewählt wird und dennoch ein Zwei-Parteiensystem existiert; Hirczy 1995).

Es lässt sich somit also festhalten, dass die technische Ausgestaltung von Wahlsystemen und die Repräsentationsprinzipien, denen sie folgen, miteinander verbun- 
Eine Typologie für die vergleichende Wahlsystemforschung

den sind, dass hier aber keinesfalls eine Deckungsgleichheit besteht. Dies gilt schon für reine Wahlsystemtypen und verstärkt sich bei der Betrachtung von Mischwahlsystemen, wie noch zu sehen sein wird. Es lohnt sich aus diesem Grund, bisherige Typologie-Ansätze genauer anzusehen.

\section{Diskussion und Problematisierung bestehender Typologie-Ansätze}

Betrachtet man nun bisherige Versuche, Wahlsysteme zu klassifizieren oder zu typologisieren, so ist André Kaiser zuzustimmen, dass dieses Feld bisher „durch ein erhebliches Maß an Konfusion und Fehlklassifikation gekennzeichnet" (2002: 1549 ) ist. Dies liegt zum einen daran, dass unterschiedliche Versuche auf verschiedenen Grundlagen basieren; zum anderen verschärft sich das Problem im Falle asymmetrischer Definitionen, bei denen die Mehrheitswahl nach deren Entscheidungsregel, die Verhältniswahl jedoch nach deren Repräsentationsprinzip eingeordnet wird (Nohlen 2009 a: 134). Die bestehenden Typologie-Ansätze, die mit dem letztgenannten Problem nicht konfrontiert sind, haben im Wesentlichen gemeinsam, dass sie sich entweder auf die Dimension des Repräsentationsprinzips eines Wahlsystems oder auf die Dimension der technischen Ausgestaltung beziehen. Diese Ansätze sollen im Folgenden kritisch dargestellt werden.

Nohlen (1984, 2009 a: 130 ff.) sieht das Repräsentationsprinzip als zentrales Definitionskriterium an und schlägt eine Einordnung von Wahlsystemen vor, die diese entweder dem Prinzip der Konzentration oder dem der Proportionalität zuordnet. Diese dichotome Sichtweise erlaubt zwar eine zweckgebundene technische Mischung von Wahlsystemen, sieht diese aber nicht als relevant für die Klassifikation von Wahlsystemen an (Nohlen 2009 a: 130 ff.). Ein Wahlsystem ist demnach entweder ein Mehrheitswahlsystem, weil es auf Konzentration abzielt, oder ein Verhältniswahlsystem, weil es auf Proportionalität abzielt, egal wie es technisch ausgestaltet ist. ${ }^{6}$ Nohlen (ebd.: 134) führt weiter aus, dass die tatsächlichen Wirkungen von Wahlsystemen nicht Teil der Definition von Wahlsystemen sein können. Dies hat neben logischen ebenfalls methodische Gründe: Eine Typologie von Wahlsystemen sollte die Möglichkeit eröffnen, auf deren Basis Wirkungen von Wahlsystemen erklären zu können. Es entsteht ein massives Endogenitätsproblem, sobald die zu erklärenden Wirkungen gleichzeitig zur Klassifikation von Wahlsystemen (also der unabhängigen Variable) dienen (Benoit 2002, 2007).

Obwohl Lijphart und Grofman (1984 b: 7) Nohlens Typologie-Ansatz ausdrücklich loben und dessen unbedingte Relevanz betonen, hat sich die englisch-sprachige

6 Eine solche dichotome Sichtweise wird von Sartori (1997: 53) geteilt. 
Literatur nahezu uneingeschränkt der technischen Dimension zugewandt, wenn es um die Klassifikation von Wahlsystemen geht. ${ }^{7}$ Hierbei besteht Einigkeit darüber, dass neben dem Stimmverrechnungsmodus selbst die Wahlkreisgröße (ggf. in Verbindung mit Sperrklauseln) das für den Einfluss auf die Zusammensetzung des Parlaments zentrale technische Element von Wahlsystemen ist. Darüber hinaus ist unumstritten, dass bei der Einordnung und Untersuchung von Wahlsystemen weitere technische Elemente (siehe die Zusammenfassung im vorangegangenen Abschnitt) zu berücksichtigen sind (Lijphart 1994; Carey/Hix 2011). Es fehlt diesen Ansätzen somit eine zentrale Variable, welche die eindimensionale Abbildung der technischen Dimension von Wahlsystemen und somit eine einfache Klassifikation erlauben würde. Trotz des Vorhandenseins mehrerer Elemente sucht man eine Komponente, die sich auf das Repräsentationsprinzip bezieht, allerdings vergeblich. Unbestritten ist, dass sich technische Elemente mischen lassen und Wahlsysteme sich somit immer auf einem Kontinuum zwischen Mehrheits- und Verhältniswahl bewegen (vgl. auch Rose 1984).

Die Gemeinsamkeit der beiden Ansätze ist also ihre jeweils eindimensionale Sichtweise, entweder auf das Repräsentationsprinzip oder auf die (allerdings in sich multi-dimensionale) technische Ausgestaltung eines Wahlsystems gerichtet. Nohlens Ansatz leidet vor allem darunter, dass er die Mischung von Repräsentationsprinzipien kategorisch ausschließt und demnach zum Beispiel nicht in der Lage ist, eine Reihe osteuropäischer Wahlsysteme eindeutig der Mehrheits- oder der Verhältniswahl zuzuordnen (Nohlen 2009 a: 238 ff.). Nohlen führt diese Systeme unter „Kombinierte Wahlsysteme“, wobei sich kombiniert auf die technische Ausgestaltung bezieht; in diesem konkreten Fall ist er Adressat seiner eigenen Kritik (s. o.), da er damit unterschiedliche Wahlsysteme auf verschiedenen Fundamenten einordnet - reine Wahlsysteme auf Grundlage des Repräsentationsprinzips, kombinierte auf Basis der technischen Ausgestaltung. Allgemeiner gesprochen besteht das Problem darin, dass sich nach Nohlen Repräsentationsprinzipien nicht mischen lassen und er dementsprechend zu dem Schluss kommt: „Electoral systems should be classified and judged in accordance with the degree to which they meet the principle of representation that they are supposed to follow. They should not be judged on whether they fulfil any of the functions of the other principle of representa-

7 Vergleiche etwa Rae 1967, Lijphart 1994, Shugart/Wattenberg 2001 b oder Gallagher/Mitchell 2005 b. 
tion" (Nohlen 1984: 88). ${ }^{8}$ Kaiser (2002: 1551) weist hingegen darauf hin, dass es „selbstverständlich“ möglich sei, Repräsentationsprinzipien zu mischen. Erst vor diesem Hintergrund ist auch die Debatte um die Frage sinnhaft, ob Mischwahlsysteme das „Beste beider Welten“ darstellen können, indem sie beide Prinzipien zufriedenstellend erfüllen bzw. zumindest darauf abzielen (Lijphart/Grofman 1984 b; Shugart/Wattenberg 2001 a; Carey/Hix 2011).

Der zweite, technische Typologie-Ansatz lässt sich vor allem in seiner Vernachlässigung der Dimension des Repräsentationsprinzips kritisieren. So erlaubt ein solcher Typologie-Ansatz keine Aussage darüber, ob die Effekte eines Wahlsystems nun dessen Zielsetzung (also dem Repräsentationsprinzip) ent- oder widersprechen. Folglich bleibt die Frage offen, ob gewisse Wahlsysteme überhaupt die Effekte intendieren, die sie verursachen. Kurzum ist eine Bewertung von Wahlsystemen ohne eine Berücksichtigung der Zielsetzung nicht möglich. Aufgrund der Kontingenz der Wirkungen eines Wahlsystems kann nicht a priori gesagt werden, welche technischen Regeln zu welchen Ergebnissen führen werden. Somit ist an dieser Stelle zunächst festzuhalten, dass das Repräsentationsprinzip und die technische Ausgestaltung die zwei verbundenen, sich jedoch keinesfalls gegenseitig determinierenden Dimensionen eines jeden Wahlsystems darstellen.

Kaiser (2002) hat in seinem höchst relevanten Beitrag bereits wegweisend auf die (nicht bloß technische) Multidimensionalität von Wahlsystemen hingewiesen. Dabei unterscheidet er zwischen der Input- und der Output-Dimension von Wahlsystemen, wobei erstere aus den technischen Regeln für die Transformation von Präferenzen in Stimmen und letztere aus den technischen Regeln für die Transformation von Stimmen in Sitze besteht. Kaiser (2002) sieht eine Mischung von Repräsentationsprinzipien insofern als möglich an, als die Regeln für die unterschiedlichen Dimensionen jeweils einem anderen Prinzip folgen können bzw. in beiden Dimensionen selbst eine Mischung vorliegen kann. Allerdings verbindet er dabei ebenfalls technische Elemente mit bestimmten Repräsentationsprinzipien und macht es dadurch schwierig, die Dynamik der Wirkungen von Wahlsystemen bei deren Kategorisierung gerecht zu werden. Insgesamt verbleibt Kaiser (2002) damit eher in der technischen Tradition von Typologie-Ansätzen.

8 Später relativiert Nohlen seine ursprüngliche Sichtweise, wenn er bei der (seiner Logik nach als Verhältniswahlsystem einzuordnenden) personalisierten Verhältniswahl bedauert, dass sie ,eine mehrheitsbildende Komponente in Form von Direktmandaten [...] gerade in einer Zeit verliert, wo diese zur Mehrheitsbildung gut würden beitragen können“ (Nohlen 2009 c: 195) und Gegnern von Überhangmandaten eine einseitige Fixierung auf Proportionalität vorwirft (Nohlen 2009 b, c). 


\section{Anforderungen an eine allgemeine Typologie von Wahlsystemen}

Aus der kritischen Diskussion bestehender Typologie-Ansätze ergeben sich konkrete inhaltliche Anforderungen an eine allgemeine Typologie von Wahlsystemen. Die zentrale Anforderung bildet die gleichzeitige Berücksichtigung der Dimensionen des Repräsentationsprinzips sowie der technischen Ausgestaltung und die damit einhergehende Überwindung eindimensionaler Betrachtungen. Diese mehrdimensionale Kategorisierung unterstreicht dabei, dass es eben keine allgemein gültige Verbindung zwischen einem Repräsentationsprinzip und bestimmten technischen Regeln gibt. Erst auf dieser Grundlage können der Gesamtcharakter eines Wahlsystems eingeschätzt und dessen Effekte bewertet werden.

Während eine Erfassung der Merkmale für die technische Dimension kein Problem darstellt, ist es weiterhin nötig, auch das Repräsentationsprinzip eines Wahlsystems a priori festzustellen. Dabei darf die Multidimensionalität der technischen Ausgestaltung eines Wahlsystems nicht unter den Tisch fallen, weil der Fokus nun nicht mehr nur auf einer Zahl von technischen Elementen liegt. Eine allgemeine Typologie von Wahlsystemen muss Spezifikationen ermöglichen, welche gerade auch die Sub-Dimensionen der technischen Dimension berücksichtigen, um Ansätzen mit verschiedenen Ziel- und Schwerpunktsetzungen einen gemeinsamen typologischen Rahmen zu liefern. Nur über eine solche Spezifikationsoption und die daraus resultierende Flexibilität lassen sich die verschiedenen Forschungsergebnisse vor einem gemeinsamen Hintergrund sinnvoll in Zusammenhang bringen (vgl. auch Jacobs/Leyenaar 2011: 508 f.). Will man beispielsweise der Frage nachgehen, welche Wirkungen welche Arten von Wahlsystemen erzielen, so wäre die Subsummierung der technischen Ausgestaltung in eine einzelne Dimension häufig unterkomplex. Parallele Mischwahlsysteme, Wahlsysteme mit einer proportionalen Verrechnungsmethode, aber einer hohen Sperrklausel, und solche mit mittelgroßen Wahlkreisgrößen würden mutmaßlich als sehr ähnlich ausgewiesen werden, bestehen aber aus völlig verschiedenen Zusammenstellungen technischer Optionen und verfolgen gegebenenfalls unterschiedliche Repräsentationsprinzipien. Die Rahmentypologie muss also eine weitere Differenzierung erlauben, ohne selbst bereits den Blick zu stark auf technische Details von Wahlsystemen zu lenken. Hierbei ist es wichtig, klar zwischen dem allgemeinen, komplexitätsreduzierenden typologischen Rahmen und dessen jeweils an eine Forschungsfrage gekoppelte Spezifikation als zwei verschiedenen Arbeitsschritten zu unterscheiden.

Neben diesen zentralen inhaltlichen Anforderungen besteht eine Reihe von methodischen Anforderungen an Typologien. Deren zusätzliche inhaltliche Konse- 
Eine Typologie für die vergleichende Wahlsystemforschung

quenzen sollen für die Entwicklung der Typologie gleichfalls leitend sein..$^{9}$ Dabei ist zunächst wichtig festzustellen, dass Typologien sich insbesondere dazu eignen, multidimensionale Phänomene abzubilden (Collier/Laporte/Seawright 2012: 227). Dabei sind sie keineswegs direkt mit der Aufgabe der Operationalisierung verbunden und sollten sich gerade nicht von Kriterien der Messbarkeit, sondern von theoretischen Aspekten leiten lassen (Pickel/Pickel 2012: 6 f.).

Das Gerüst einer Typologie bildet eine Matrix, deren Zellen die einzelnen Typen eines übergeordneten Phänomens markieren. Die definitorischen Merkmale der Zellen ergeben sich aus Zeilen- und Spaltenvariablen, den Dimensionen der Typologie. ${ }^{10}$ Die einzelnen Dimensionen können als nominale, ordinale oder kontinuierliche Variablen dargestellt werden und selbst aus mehreren Sub-Dimensionen zusammengesetzt sein (Collier/Laporte/Seawright 2008: $223 \mathrm{ff}$.). Mit Hilfe dieser Sub-Dimensionen lassen sich Typologien dann spezifizieren (vgl. auch Gerring 2012: 724 ff.). Im Falle kontinuierlicher Dimensionen helfen sogenannte polar und intermediate cases, Zelltypen zu identifizieren, ohne dabei auf willkürliche Abgrenzungen zurückgreifen zu müssen (Collier/Laporte/Seawright 2012: $222 \mathrm{f}$.).

Über die bloße Gestaltung von Typologien hinaus sehen sich zunächst rein deskriptive Herleitungen von Typologien mit der Zielsetzung konfrontiert, einen Erkenntnisfortschritt in einer sozialwissenschaftlichen Welt zu liefern, die vor allem auf das Auffinden kausaler Zusammenhänge ausgerichtet ist. Gerring (2012: 741) führt aus, dass ,true innovation in descriptive inference is established only by delineating a fundamentally novel empirical terrain, or by thoroughly revising our sense of an established terrain". Gleichzeitig muss aber betont werden, dass die Untersuchung kausaler Zusammenhänge existentiell davon abhängt, dass empirische Phänomene adäquat beschrieben beziehungsweise eingeordnet werden: ,[C]lassifications remain the requisite, if preliminary, condition for any scientific discourse" (Sartori 1970: 1040; vgl. auch Collier/Laporte/Seawright 2008: 162), was sicher gleichermaßen für Typologien als mehrdimensionale Klassifikationen gilt. Vor diesem Hintergrund wird klar, dass Typologien eine zentrale Rolle im Forschungsprozess einnehmen können beziehungsweise, nach Sartori (1970), sogar müssen. Um diese Rolle einzunehmen, sollte eine Typologie gleichzeitig Grundlage für Erklärungen sein und Hinweise auf zu Erklärendes geben. Dies geschieht im Wesentlichen dadurch, dass die Zelltypen sowohl als abhängige wie auch als un-

9 Für intensive Auseinandersetzungen mit dem methodischen Werkzeug der Typologie siehe Collier/ Laporte/Seawright 2008, 2012; vgl. auch Sartori 1970.

10 Mit Blick auf unsere Typologie stellen leere Zellen in diesem Zusammenhang kein Problem dar, da die theoretische Bedeutung der Dimensionen eine zumindest theoretische Relevanz aller Zelltypen bedingt. 
abhängige Variable fungieren können (Collier/Laporte/Seawright 2012: 226). Von großer Bedeutung ist schließlich die strukturierende Leistung der Typologie, welche, von konkreter Messung zunächst unabhängig, einen Rahmen schafft, in dem sich etwa Forschungsergebnisse von Einzelfallstudien mit denen von komparativquantitativen Studien vergleichen und verbinden lassen (Collier/Laporte/Seawright 2008: $162 \mathrm{ff}$.).

Der konzeptionelle Mehrwert der multidimensionalen Betrachtung kann hier bereits anhand zweier zentraler Themen innerhalb der Wahlsystemforschung skizziert werden.

- Wahlsystemwandel: Der strukturierende Beitrag der Typologie zur Untersuchung des Wandels bzw. der Genese von Wahlsystemen besteht in erster Linie darin, aufzuzeigen, dass eine Entscheidung für ein bestimmtes Repräsentationsprinzip keinesfalls die technische Ausgestaltung eines Wahlsystems determiniert. Darüber hinaus wird bei der Betrachtung beider Dimensionen deutlich, dass Reformdebatten sowohl das Repräsentationsprinzip in Verbindung mit der technischen Ausgestaltung als auch eine Anpassung der technischen Elemente bei konstantem Repräsentationsprinzip betreffen können. Folglich kann Wandlungsdruck zum einen entstehen, indem technische Regeln Ergebnisse produzieren, die nicht mehr mit dem Repräsentationsprinzip in Einklang stehen. In diesem Fall wäre der potenzielle Wandel in einer technischen Anpassung bei Beibehaltung des Repräsentationsprinzips begründet. Zum anderen kann Wandlungsdruck aus einer Diskrepanz zwischen dem Repräsentationsprinzip des aktuellen Wahlsystems und der Vorstellung relevanter Akteure darüber, was das Wahlsystem zu leisten habe, entstehen. Diese Diskrepanz kann sowohl machtpolitisch als auch durch soziopolitischen Wandel in der Gesellschaft bedingt sein (Massicotte 2005; LeDuc 2011).

- Mischwahlsysteme: Die differenzierte Sichtweise verdeutlicht, dass eine Mischung sich auf das Repräsentationsprinzip, auf die technische Gestaltung oder auf beides beziehen kann. Während dies die Diskussion rund um diese Systeme zunächst zu verkomplizieren scheint, ist jedoch genau das Gegenteil der Fall. So werden gerade Diskussionen, bei welchen Mischwahlsysteme auf der einen Seite wegen ihrer Zielsetzung, auf der anderen aber auf Basis ihrer technischen Gestaltung als solche eingeordnet werden, dazu gebracht, sich in den allgemeinen typologischen Rahmen einzuordnen und somit von asymmetrischen Vergleichen Abstand zu nehmen. Über die Betrachtung des typologischen Rahmens kann weiterhin differenziert analysiert werden, ob technisch gemischte Wahlsysteme aus einer Mischung von Repräsentationsprinzipien resultieren oder aber eindeutig mit einem Repräsentationsprinzip verbunden sind und aufgrund sozio-politi- 
Eine Typologie für die vergleichende Wahlsystemforschung

scher Rahmenbedingungen technisch weit weniger eindeutige Regeln erhalten haben. So geht die mehrdimensionale Betrachtung von Wahlsystemen mit einer Erhöhung der potenziellen Erklärungen nicht nur für bestimmte Wirkungen, sondern auch für bestimmte Gestaltungsentscheidungen einher. Mithilfe der Hinzunahme des Repräsentationsprinzips eines Wahlsystems wird die Debatte, ob Mischwahlsysteme das „Beste beider Welten“ hervorbringen, auch vor die Frage gestellt, inwiefern die Erreichung dieses vermeintlich „Besten beider Welten“ überhaupt ein Ziel des Wahlsystems darstellt.

\section{Entwurf einer Typologie bei gleichzeitiger Berücksichtigung von Reprä- sentationsprinzip und technischer Ausgestaltung}

\section{a) Konstruktion eines allgemeinen typologischen Rahmens}

Abbildung 1 zeigt den von uns vorgeschlagenen allgemeinen typologischen Rahmen. Die Matrix zeigt die beiden relevanten Dimensionen „Repräsentationsprinzip“ und ,technische Ausgestaltung" und bezeichnet verschiedene Typen von Wahlsystemen. Die Pfeile unter bzw. neben den Dimensionsbezeichnungen zeigen an, dass beide Dimensionen kontinuierlich sind - das Repräsentationsprinzip kann einseitig sein oder aus einer (gewichteten) Balance beider Prinzipien bestehen; genauso bewegen sich Wahlsysteme technisch zwischen einer reinen Mehrheitswahl und einer reinen Verhältniswahl. Die Zelltypen eins bis neun zeichnen sich durch eine jeweils spezifische Kombination der Ausprägungen in den beiden Dimensionen aus, wobei diese Ausprägungen im Sinne von intermediate und polar zu verstehen sind und eine Spezifikation der Abgrenzungen erst mit der Beantwortung einer bestimmten Forschungsfrage vorgesehen ist.

Zunächst ist über die Berücksichtigung der beiden Dimensionen gewährleistet, dass der über den technischen Bereich hinausgehenden Multidimensionalität von Wahlsystemen Rechnung getragen wird. Da die technische Dimension zudem zunächst als Kontinuum bestehend aus einer Reihe von Sub-Dimensionen dargestellt wird, ist die Typologie auch allgemein. Alle theoretisch möglichen sowie empirisch vorkommenden Wahlsysteme können in die Typologie eingeordnet werden. Sogenannte „Residualkategorie[n]“ (Nohlen 2009 a: 130) werden damit verhindert - gerade die zunehmende Häufigkeit von Mischwahlsystemen wird somit auch typologisch berücksichtigt. Im Vergleich zu Nohlen (2009 a) gelingt es auch, die Vielzahl osteuropäischer Wahlsysteme mit technischer Mischung direkt in der Typologie zu verorten. Die multidimensionale Einordnung von Wahlsystemen erlaubt es folglich 
Abbildung 1: Allgemeine Typologie von Wahlsystemen

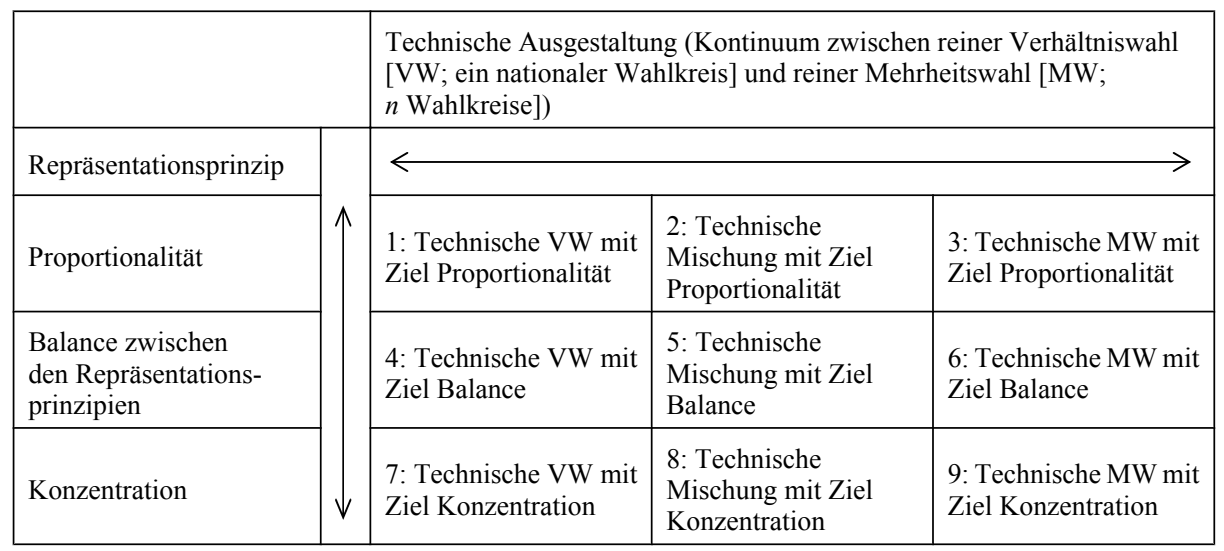

Quelle: Eigene Darstellung.

ebenfalls, in späteren Analysen zwischen intendierten und nicht-intendierten Effekten von Wahlsystemen zu unterscheiden. Gerade so können auch auf den ersten Blick widersinnige technische Regeln auf ihre vielleicht überlegene Wirkung hinsichtlich ihres Repräsentationsprinzips überprüft werden. Generell wird es möglich, zwischen technisch identischen Wahlsystemen zu unterscheiden, sofern diese unterschiedliche Repräsentationsprinzipien verfolgen, sowie festzustellen, inwiefern die unterschiedliche Wirkung technisch ähnlicher Wahlsysteme auf absichtsvolles Design oder auf die unvorhergesehenen Interaktionseffekte zwischen sozio-politischen Rahmenbedingungen und Wahlsystem zurückzuführen sind. Die Zelltypen geben Anlass zur Untersuchung genau solcher Fragestellungen und bieten aus methodologischer Sicht größeres Potenzial als bloß technisch geleitete Einordnungen.

Der vorgeschlagene typologische Rahmen verbindet Einzelfallstudien und vergleichende Studien erstens natürlich insofern, als er beiden eine gemeinsame Grundlage für die Analyse von Wahlsystemen liefert und Forschungsergebnisse damit verknüpfbar macht. Zweitens verbindet die hier vorgenommene zweidimensionale Betrachtung diese sich vor allem in der Fallzahl, weniger in den Fragestellungen unterscheidenden Studien aber auch, indem zwei Forschungsrichtungen zusammengeführt werden. Während komparative Studien häufig quantitativ angelegt sind und sich vor allem in der Herausarbeitung der Wirkungen der technischen Ausgestaltung hervorgetan haben, sind es bisher exklusiv Einzelfallstudien, die gezielt (wenn auch nicht immer explizit) die Frage nach dem Repräsentationsprinzip eines Wahlsystems stellen (vgl. etwa die Arbeiten in Gallagher/Mitchell 2005 a; Nohlen 2009 a). Insofern besteht der Mehrwert dieses Typologie-Ansatzes nicht bloß in der 
Rahmung der verschiedenen Ansätze, sondern auch in der Zusammenführung deren inhaltlicher Stärken.

Die Typologie bietet weiterhin die notwendige Flexibilität, indem die Dimensionen je nach Forschungsfrage und theoretischen Implikationen spezifiziert werden können. Hierin besteht genau die komplexitätsreduzierende Rahmungsfunktion, die von einer allgemeinen Typologie erwartet wird. Gerade die technische Multidimensionalität von Wahlsystemen wird dabei keineswegs vernachlässigt, sondern kann mithilfe einer Ausdifferenzierung der technischen Dimension über Sub-Dimensionen abgebildet werden. Wie sich das Kontinuum der technischen Dimension mittels einiger Sub-Dimensionen abbilden lässt, zeigen wir im folgenden Abschnitt. Dort nutzen wir die Rahmungsfunktion der allgemeinen Typologie, erhöhen dabei jedoch den Detailgrad unserer Betrachtung.

\section{b) Berücksichtigung technischer Multidimensionalität}

Wie bereits erläutert, muss die im obigen Rahmen dargestellte technische Dimension als aus diversen technischen Sub-Dimensionen bestehend begriffen werden.

Eine Vielzahl technischer Elemente kann dafür sorgen, dass ein Wahlsystem rein technisch eher eine Verhältnis- bzw. eine Mehrheitswahl ist. Elemente, welche für das Kontinuum zwischen technischer Mehrheits- und technischer Verhältniswahl nicht zentral sind, betreffen vor allem die Stimmgebung und die Form der Kandidatur: Stimmen Wähler für einzelne Personen oder Listen? Können sie Listen verändern, verknüpfen oder eigene Listen erstellen? Die Antworten auf diese Fragen unterscheiden Wahlsysteme voneinander und sind grundsätzlich relevant. Für die Einordnung von Wahlsystemen als Verhältnis- oder Mehrheitswahlsystem spielen sie aber keine Rolle und werden daher im Weiteren nicht berücksichtigt. Für das Kontinuum zentral sind vor allem Elemente, welche Stimmenverrechnung und Wahlkreiszuschnitt betreffen. Abbildung 2 zeigt im Sinne von polar und intermediate cases (siehe Abschnitt 4) für jedes dieser technischen Elemente an, bei welchen Ausprägungen es aus technischer Perspektive zu einer reinen Mehrheitswahl, einer reinen Verhältniswahl bzw. zu einer technischen Mischung führt. Der Pfeil skizziert, dass es sich wie schon in Abbildung 1 auch hier um kontinuierliche Variablen handelt. Das Design eines Wahlsystems betreffend, lassen sich diese technischen Elemente theoretisch in jeglichen Ausprägungskombinationen miteinander kombinieren. 
Abbildung 2: Technische Sub-Dimensionen

\begin{tabular}{|c|c|c|c|}
\hline Technisches Element & $\begin{array}{l}\text { Technische } \\
\text { Mehrheitswahl }\end{array}$ & Technische Mischung & $\begin{array}{l}\text { Technische } \\
\text { Verhältniswahl }\end{array}$ \\
\hline & \multicolumn{3}{|c|}{$\longleftrightarrow$} \\
\hline Verrechnungsmodus & $\begin{array}{l}\text { Relative bzw. } \\
\text { absolute Mehrheit } \\
\text { der Stimmen im } \\
\text { Wahlkreis }\end{array}$ & $\begin{array}{l}\text { Verwendung beider Modi, } \\
\text { etwa in Grabenwahlsyste- } \\
\text { men }\end{array}$ & $\begin{array}{l}\text { Proportionale Me- } \\
\text { thoden (z. B. d'Hondt, } \\
\text { Single Transferable } \\
\text { Vote) }\end{array}$ \\
\hline Wahlkreisgröße & 1 & $\begin{array}{l}\text { Mindestens ein Wahlkreis } \\
\text { der Größe } k \text { mit } 1<k<n\end{array}$ & $\begin{array}{l}\text { Gleicht Parlaments- } \\
\text { größe } n\end{array}$ \\
\hline Gesetzliche Sperrklausel & Hoch & Moderat & Keine \\
\hline $\begin{array}{l}\text { Kompensation durch } \\
\text { zusätzliche tiers }\end{array}$ & Keine Kompensation & $\begin{array}{l}\text { Mindestens zweites tier, } \\
\text { keine vollständige Kom- } \\
\text { pensation }\end{array}$ & $\begin{array}{l}\text { Vollständige Kompen- } \\
\text { sation }\end{array}$ \\
\hline
\end{tabular}

Quelle: Eigene Darstellung.

Der Verrechnungsmodus der Stimmen ist ein Element der technischen Ausgestaltung, auf das häufig alleine abgehoben wird, wenn es gilt, Wahlsysteme einzuordnen. Hierbei wird zwischen Modi der Mehrheitswahl, wie absolute und relative Mehrheitswahl oder auch dem Alternative Vote als Spezialfall der absoluten Mehrheitswahl, einerseits und Methoden der Verhältniswahl (d'Hondt, Sainte-Laguë, Hare-Niemeyer, Single Transferable Vote und weiteren) ${ }^{11}$ andererseits unterschieden. Jenseits dieser Stimmverrechnungsmodi sind theoretisch auch Methoden denkbar, die eine Mischung der reinen Typen darstellen; diese sind praktisch allerdings nicht relevant. Nichtsdestotrotz ist eine Mischung der reinen Typen denkbar, wenn - wie in Grabenwahlsystemen - ein Teil der Sitze nach Methoden der Mehrheitswahl, ein anderer Teil nach Methoden der Verhältniswahl vergeben wird. Je nach Größe der jeweiligen Anteile tendiert die Mischung eher Richtung Mehrheits- oder Richtung Verhältniswahl.

Dem technischen Element „Wahlkreisgröße“ hat sich insbesondere Lijphart (1994) ausführlich gewidmet. Je kleiner ein Wahlkreis, desto höher ist ceteris paribus der Stimmanteil für Wahlbewerber, der nötig ist, um einen Sitz zu erreichen. Lijphart nennt dies den effective threshold. Es ist leicht vorstellbar, dass Wahlsys-

11 Dass sich auch die Verfahren der reinen Wahlsystemtypen hinsichtlich ihrer konzentrierenden bzw. proportionalitätserhaltenden Wirkung unterscheiden, sei der Vollständigkeit halber erwähnt. So gilt die relative Mehrheitswahl als stärker konzentrierend als die absolute, oder d'Hondt als stärker konzentrierend als Sainte-Laguë. Im Vergleich zu den Unterschieden zwischen den Wahlsystemtypen sind diese Nuancen jedoch nicht von zentraler Bedeutung. 
Eine Typologie für die vergleichende Wahlsystemforschung

teme mit sehr kleiner Wahlkreisgröße nahe 1 auch unter der Verwendung von Verrechnungsverfahren der Verhältniswahl Mehrheitswahlsystemen näherkommen. Bei einer Wahlkreisgröße von 2 etwa erhalten entweder die beiden stärksten Parteien je einen Sitz oder die stärkste Partei beide. Bei einer Wahlkreisgröße von 1 verschmelzen alle Verrechnungsmodi, egal ob solche der Verhältnis- oder der Mehrheitswahl, in einem reinen Mehrheitswahlsystem. Je nachdem, ob die Wahlkreisgröße $k$ näher bei 1 oder näher bei $n$ liegt, entspricht die technische Ausgestaltung eher der Mehrheits- oder eher der Verhältniswahl. Vorsicht ist allerdings geboten, die Variable „Wahlkreisgröße“ als „durchschnittliche Wahlkreisgröße“ zu verkürzen. Dies kann für bestimmte Studien hilfreich sein; allgemein gilt jedoch, dass für ein System mit 20 Fünfer-Wahlkreisen andere Ergebnisse zu erwarten sind als für ein System mit 19 Einer-Wahlkreisen und einem Wahlkreis der Größe 81. Die Variable „Wahlkreisgröße" muss daher in ihrer allgemeinsten Form die ganze Struktur der Wahlkreisgrößen beinhalten.

Neben einer effektiven Hürde, die über die Wahlkreisgröße generiert wird, sind auch formal verankerte Hürden in der Praxis von Wahlsystemen relevant. Die Effekte einer formalen Hürde sind vom Grundsatz her die gleichen wie die einer effektiven: Je höher eine solche Hürde ist, desto eher trägt ein Wahlsystem Züge der Mehrheitswahl, je geringer sie ist, desto näher kommt das Wahlsystem einem Verhältniswahlsystem. Ist eine Hürde so niedrig, dass sie keine Auswirkungen besitzt, oder existiert gar keine formale Hürde, so verschmilzt ein Wahlsystem hinsichtlich dieses Aspekts mit der Reinform der Verhältniswahl. Ist die Hürde hingegen so hoch, dass es nur einer oder zwei Parteien gelingt, diese zu überspringen, so verschmilzt das Wahlsystem mit dem Typus der Mehrheitswahl. Dazwischen sind alle möglichen Abstufungen denkbar.

Als letztes zu diskutierendes Element schließlich ist in Abbildung 2 das Vorhandensein mehrerer Ebenen der Stimmverrechnung (tiers) aufgeführt, die über einen Kompensationsmechanismus verbunden sind. Wenngleich kompensatorische Mischwahlsysteme Grabenwahlsystemen vordergründig stark ähneln, ist hier eine Differenzierung vorzunehmen. Zwar haben beide Typen gemein, dass Stimmen in unterschiedlicher Weise auf mindestens zwei Ebenen verrechnet werden und dass beide Ebenen sich in der Regel hinsichtlich des Modus der Stimmverrechnung unterscheiden. Der zentrale Unterschied besteht allerdings darin, dass sich in Grabenwahlsystem - wie oben beschrieben - durch das Nebeneinander der Ebenen eine Mischung der Stimmverrechnungsverfahren erreichen lässt. Dies ist in kompensatorischen Systemen nicht zwingend der Fall. Sobald die Ebene der technischen Verhältniswahl die Ebene der technischen Mehrheitswahl vollständig kompensiert, verschmilzt ein solches System mit dem System der reinen Verhältniswahl - auch 
wenn Ebenen der technischen Mehrheitswahl vorhanden sind und anders als in Grabenwahlsystemen. Findet keinerlei Kompensation statt, so ist das Wahlsystem hinsichtlich dieses Elements als Mehrheitswahl einzuordnen; findet eine teilweise Kompensation statt, liegt eine technische Mischung vor.

Während man für jedes einzelne Element losgelöst vom übrigen Wahlsystem dessen Funktion abschätzen kann, ist es jedoch von großer Bedeutung, ein Wahlsystem immer als Zusammenwirken diverser technischer Elemente zu betrachten. So ist die Wahlkreisgröße auf einer unteren Ebene egal, wenn auf der oberen Ebene ohnehin eine Vollkompensation stattfindet; bei einer Teilkompensation hingegen spielt sie eine Rolle. Genauso hat eine relativ niedrige oder auch moderat hohe Sperrklausel in einem Ein-Personen-Wahlkreis keinerlei Effekt, wirkt sich jedoch stark auf Ergebnisse aus, sobald die Größe des Wahlkreises zunimmt. Diese Beispiele des Zusammenspiels verschiedener technischer Elemente verdeutlichen die Notwendigkeit, alle Elemente gemeinsam zu berücksichtigen. Gleichzeitig muss jedoch der Blick auf die wesentlichen Dimensionen eines Wahlsystems gerichtet werden. Der Fokus auf konkrete Fragen braucht beides, die passende Spezifikation der technischen Dimension sowie die Berücksichtigung des Repräsentationsprinzips. Nur auf diesem Wege lassen sich Design-Entscheidungen beurteilen und Wirkungen von Wahlsystemen verstehen. Die im folgenden Abschnitt vorzunehmende empirische Einordnung konkreter Fallbeispiele soll die Sinnhaftigkeit dieses Vorgehens verdeutlichen.

\section{Einordnung konkreter Fallbeispiele}

Verfolgt man nun in einem weiteren Schritt die Absicht, die Typologie durch die Einordnung konkreter Wahlsysteme mit Leben zu füllen, so lassen sich die Wahlsysteme nicht per se, sondern nur im Kontext der Länder, in denen sie Anwendung finden, beurteilen. Dies liegt weniger an der technischen Ausgestaltung als vielmehr am Repräsentationsprinzip, das den Wahlsystemen selbst nicht (oder zumindest nicht direkt) entnommen werden kann. Hierbei zeigt sich eine generelle Schwierigkeit: Obwohl theoretisch von hoher Bedeutung, ist die Einordnung des Repräsentationsprinzips eines Wahlsystems mit einigen Unwägbarkeiten verbunden. In den seltensten Fällen wird offiziell festgehalten, welches Hauptziel ein konkretes Wahlsystem erreichen soll. ${ }^{12}$ Dementsprechend orientiert sich unsere Einordnung verschiedener Wahlsysteme im Hinblick auf deren Repräsentationsprinzipien zum einen an den jeweiligen Verfassungstexten (Nohlen 2009 a: 145) sowie an Exper-

12 Gegenbeispiele sind etwa Irland oder die Niederlande, die in den Verfassungsartikeln 16 bzw. 50 explizit festlegen, dass das Wahlsystem der Logik proportionaler Repräsentation zu folgen hat. 
Eine Typologie für die vergleichende Wahlsystemforschung

teneinschätzungen (wie sie etwa in der Form von Einzelfallstudien vorhanden sind; siehe Gallagher/Mitchell 2005 a). Konkret stellen wir jeweils die Frage, was die Designer eines Wahlsystems bzw. diejenigen, die es zuletzt einer Reform unterzogen haben, in Bezug auf das Repräsentationsprinzip im Sinn hatten. Dies bedeutet gleichzeitig einen klaren methodischen Fortschritt gegenüber nicht näher spezifizierten Gesamteinschätzungen eines Wahlsystems, allerdings auch die Herausforderung, die relevanten Akteure und deren Intentionen möglichst eindeutig festzustellen.

Die in Tabelle 1 aufgelisteten Fallbeispiele bieten eine Auswahl an Wahlsystemen, welche bei der für jedes Land jeweils letzten Wahl Anwendung fanden. Diese ordnen wir, basierend auf der in Abschnitt 5.b) vorgestellten Spezifikation, konkret in unsere Typologie ein. Die Fallauswahl erfolgt zum einen auf Basis des Vorhandenseins einer hinreichenden Grundlage für die Zuordnung des Repräsentationsprinzips, zum anderen ist sie so angelegt, dass sie sowohl für die Dimension des Repräsentationsprinzips als auch für die technische Ausgestaltung die volle Bandbreite an Möglichkeiten abdeckt. Durch diese Breite kann der Mehrwert der vorgeschlagenen Typologie an den ausgewählten Beispielen verdeutlicht werden.

Großbritannien und Kanada besitzen Wahlsysteme, die jeweils eindeutig mit dem Repräsentationsprinzip der Konzentration verbunden sind (Farrell 2011: 13 ff.; Massicotte 2005). Die Reformdebatten in beiden Ländern zeigen, dass insbesondere die Konzentration des Parteiensystems durch das aktuelle Wahlsystem als gewichtiges Gegenargument gegen Reformvorschläge vorgebracht wird (Dunleavy/Margetts 1995; Jenkins Commission 1998: 53 ff.; Massicotte 2005: 111). Ein Auszug aus einem Gerichtsurteil des obersten Gerichtshofs zum Wahlkreiszuschnitt in der kanadischen Provinz Saskatchewan unterstreicht diese Einschätzung: „Deviations from equality will be permitted where they can be justified as contributing to the better government of the people as a whole" (Supreme Court 1991). Litauen und Spanien sind Beispielfälle für Wahlsysteme, die mit dem Ziel einer Balance beider Prinzipien entworfen wurden. Hierfür waren jeweils die Verhandlungen und Kompromisse zwischen alten und neuen Eliten verantwortlich. Aus diesen ging hervor, dass das Wahlsystem sowohl eine proportionale als auch eine konzentrierende Funktion erfüllen sollte (Benoit 2007: 380 ff.; Hopkin 2005: 375 ff.). Irland, Israel und die Niederlande haben jeweils Wahlsysteme, denen klar das Repräsentationsprinzip der Proportionalität zugeordnet werden kann - dies ist so einerseits (in den Fällen Irland und Niederlande, siehe Fußnote 12) aus den Verfassungstexten zu entnehmen sowie andererseits klar aus der Entstehungsgeschichte der Wahlsysteme abzulesen (Gallagher 2005: 512 ff.; Andeweg 2005; Rahat/Hazan 2005: 334 ff.). 


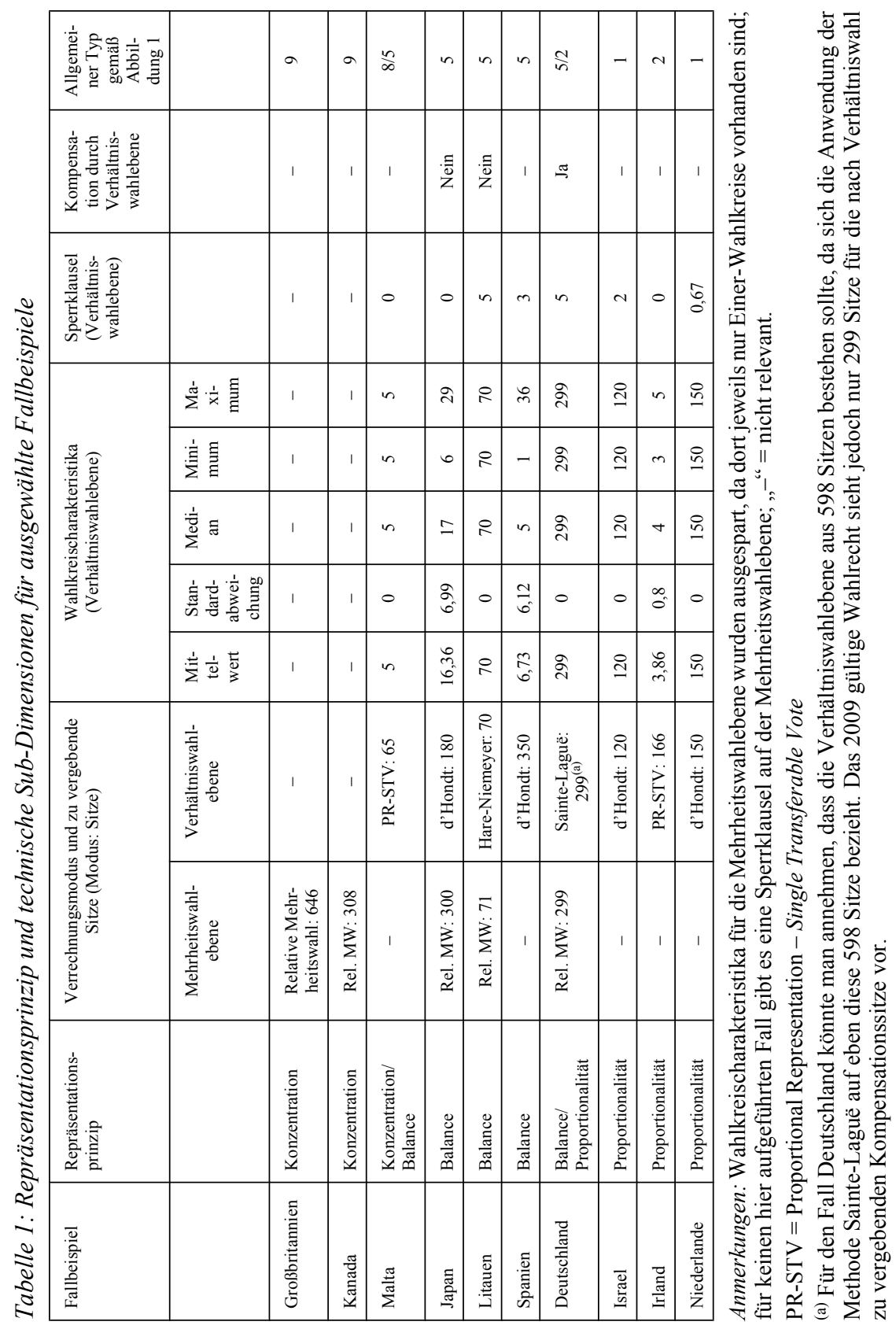


Eine Typologie für die vergleichende Wahlsystemforschung

Neben diesen klaren Fällen sind Japan, Malta und Deutschland gute Beispiele für Unwägbarkeiten, die bei der Einschätzung des Repräsentationsprinzips auftreten können. In Japan wurde die Reform des Wahlsystems Anfang der neunziger Jahre hauptsächlich durch Korruptionsskandale ausgelöst. Dabei spielten konkrete Zielsetzungen für das Wahlsystem eine zunächst nachgeordnete Rolle. Gleichwohl flossen sowohl die Weigerung der ehemaligen Oppositionsparteien, ein reines Mehrheitswahlsystem zu unterstützen, als auch das Ziel der Generierung eines Zwei-Parteien-Systems in die Entwicklung des neuen Wahlsystems ein (Reed 2005: 281; Sakamoto 1999: 428 ff.). Wir ordnen Japan daher als Fall mit ausbalanciertem Repräsentationsprinzip ein.

Auch aus dem Ausbleiben von Wahlsystemreformen können Schlüsse auf das Repräsentationsprinzip gezogen werden: Das maltesische Wahlsystem wurde 1976 gerade deshalb keiner Reform unterzogen, weil trotz seiner technischen Ausgestaltung mit bedeutenden Verhältniswahlelementen das maltesische Parteiensystem konzentriert war (Proctor 1980: 318 ff.; Hirczy 1995: 260 f.). Da nicht gesichert ist, inwiefern die Proportionalitätsleistung des Wahlsystems in die Entscheidung gegen eine Reform eingeflossen ist, lässt sich im Falle Maltas weder eindeutig das Prinzip der Konzentration noch das Ziel einer Balance zuordnen (vgl. Tab. 1).

Ebenfalls strittig ist die Einordnung des deutschen Wahlsystems. Während auf der einen Seite das Ziel der Proportionalität hervorgehoben wird, ${ }^{13}$ wird auf der anderen Seite gerade mit Blick auf den Entwurf des Wahlsystems auch das Ziel einer gewissen Konzentration des Parteiensystems als einer Lehre aus Zeiten der Weimarer Republik betont (Strohmeier 2009: 14 ff.; Jesse 2009: 114 ff.; Saalfeld 2005: 210 f.). Weiterhin hat das Bundesverfassungsgericht in seinen Entscheidungen zum Wahlsystem und dessen Reformen immer wieder dessen Verhältniswahlcharakter hervorgehoben (Nohlen 2009c), gleichzeitig aber auch auf die wünschenswerte Konzentrationsfunktion eines Wahlsystems hingewiesen (BVerfG 1997). Demnach kann zwar festgehalten werden, dass dem Ziel der Proportionalität ein größeres Gewicht beigemessen wird, jedoch eine Erfüllung beider Repräsentationsprinzipien ausdrücklich erwünscht ist. Hinsichtlich der Dimension des Repräsentationsprinzips weist der Fall des deutschen Wahlsystems klar darauf hin, dass diese kontinuierlich ist und zwischen den Polen Konzentration und Proportionalität verschiedene Gewichtungen dieser Ziele nicht nur theoretisch denkbar, sondern auch praktisch bedeutsam sind.

Die zusätzliche Betrachtung der konkreten technischen Ausgestaltungen der Wahlsysteme unterstreicht die Notwendigkeit einer multidimensionalen Betrach-

13 Siehe etwa Prittwitz 2011, Strohmeier 2009: 17, Nohlen 2009 a oder Schoen 2007: 863. 
tung. Bei der Darstellung der technischen Ausgestaltung von Wahlsystemen orientieren wir uns an Abbildung 2. Hierbei entscheiden wir uns bewusst dagegen, jedes einzelne technische Detail in den vergleichenden Überblick mit aufzunehmen, sind aber präzise genug, um sämtliche Stellschrauben eines trade-offs zwischen Verhältnis- und Mehrheitswahl zu erfassen. Tabelle 1 listet in diesem Sinne Informationen zu allen relevanten technischen Elementen auf und umfasst neben dem Repräsentationsprinzip die folgenden technischen Sub-Dimensionen: Verrechnungsmodi inklusive der Sitzanteile der verschiedenen Ebenen, Charakteristika der Wahlkreisgröße, Höhe der gesetzlichen Sperrklausel sowie Art und Weise der Kompensationsverbindung zwischen verschiedenen Ebenen. Wir verbleiben bei der Logik kontinuierlicher Dimensionen sowie intermediate und polar cases, indem wir auf eine willkürliche Festlegung von Schwellenwerten, welche etwa technische Typen voneinander abgrenzen, verzichten. An dieser Stelle geht es vor allem darum, den Mehrwert der Typologie nachzuweisen und nicht darum, eine Diskussion über exakte Schwellenwerte zu führen.

Das Repräsentationsprinzip Konzentration scheint zunächst relativ klar mit einer technisch reinen Mehrheitswahl verbunden zu sein. Großbritannien und Kanada sind Beispiele für diesen Zusammenhang: In den Wahlsystemen beider Staaten gibt es ausschließlich Einer-Wahlkreise, aus deren Siegern sich die Parlamente zusammensetzen. Hinsichtlich der Entscheidungsregel wie auch der Wahlkreisgröße herrscht hier also die reinste Form der technischen Mehrheitswahl vor. Da zudem keine zweite Ebene existiert, ist die Frage nach Kompensationen obsolet, und das Fehlen einer formalen Hürde ist ohne Bedeutung. Beide Staaten können somit als klassische Fälle des allgemeinen Typs 9 gelten, in denen die technische Mehrheitswahl das Repräsentationsprinzip der Konzentration verfolgt. Allerdings zeigen genau diese Beispiele, dass, während in Großbritannien nach nahezu allen Wahlen auf stabilen Mehrheiten beruhende Ein-Parteien-Regierungen zustande gekommen sind, dies für Kanada nicht der Fall ist. Das kanadische Wahlsystem erfüllt das Ziel der Konzentration daher nicht oder nur bedingt, da dort Ein-Parteien-Regierungen typischerweise ,auf Zeit spielende“ Minderheitsregierungen sind (Massicotte 2005: 110 ff.). Nur durch eine saubere Trennung zwischen Repräsentationsprinzipien und empirisch beobachtbaren Effekten von Wahlsystemen kann jedoch Fragen nachgegangen werden, die sich mit der Evaluierung von Wahlsystemen befassen. Eine solche Trennung können eindimensionale, entweder allein auf eine Gesamtwirkung des Wahlsystems oder dessen technischen Charakter fokussierte Typologien (siehe Abschnitt 3) nicht leisten.

Am Beispiel Malta lässt sich aufzeigen, dass der Wunsch einer konzentrierenden Wirkung des Wahlsystems keinesfalls zwingend mit der technischen Mehrheitswahl 
Eine Typologie für die vergleichende Wahlsystemforschung

verbunden sein muss. Die Entscheidungsregel Single Transferable Vote ist eine klassische Stimmverrechnungsmethode der Verhältniswahl. Auch eine gesetzliche Sperrklausel gibt es nicht. Eine konzentrierende Wirkung wird allerdings durch die relativ geringe Wahlkreisgröße (fünf) erzielt, über die landesweit keine Varianz besteht und deren Effekt durch keine zweite Ebene mit größeren Wahlkreisen kompensiert wird. Technisch gesehen ist das maltesische Single Transferable Vote somit ein Mischwahlsystem, und Malta ist, da das Repräsentationsprinzip nicht eindeutig bestimmt werden kann, insgesamt als Typ 5 oder 8 einzuordnen. Eindimensionale Typologien scheitern für den Fall Malta entweder an ihrem Indeterminismus (das Wahlsystem wirkt insgesamt sowohl proportional als auch konzentrierend) oder daran, dass eine technische Mischung mit dem Vorhandensein zweier Ebenen verknüpft und Malta somit nicht berücksichtigt wird (Shugart/Wattenberg 2001 a).

Der Fall Malta lenkt unseren Blick zudem auf die empirisch nicht vorhandenen allgemeinen Typen 3, 4, 6 und 7 und macht deutlich, dass unter bestimmten soziopolitischen Rahmenbedingungen das Ziel der Konzentration oder zumindest einer Balance der Prinzipien durchaus sinnvollerweise mithilfe einer technischen Verhältniswahl (Typen 4 und 7) erreicht werden kann. Ebenfalls, wenn auch ohne empirisch verwandten Fall, könnten die Typen 3 und 6 (technische Mehrheitswahl mit Repräsentationsprinzip Proportionalität bzw. Balance) erfolgreich dort funktionieren, wo sich etwa in verschiedenen Regionen jeweils nur zwei Parteien gegenüberstehen.

Wahlsysteme, die unter der Prämisse entworfen bzw. reformiert wurden, möglichst beide Repräsentationsprinzipien zu erfüllen, sind etwa Spanien, Japan und Litauen. Technisch sind in Litauen und Japan Zwei-Ebenen-Mischwahlsysteme vorhanden. Da keine Kompensation zwischen den Ebenen erfolgt, sondern die Entscheidungsregeln relative Mehrheitswahl und d'Hondt bzw. Hare-Niemeyer separat voneinander angewendet werden, handelt es sich konkret um Grabenwahlsysteme. Der Anteil der Verhältniswahlebene beträgt 37,5 (Japan) bzw. rund 50 Prozent (Litauen). Die Verhältniswahlebene selbst unterliegt in beiden Fällen weiteren Einschränkungen. Während das japanische Wahlsystem seine Verhältniswahlebene in Mehrpersonenwahlkreise aufteilt und dadurch die effektive Sperrklausel erhöht wird, besteht das litauische aus einem einzigen nationalen Wahlkreis. Für diesen ist dann jedoch eine relativ hohe Fünf-Prozent-Sperrklausel vorgesehen. Spaniens Wahlsystem hingegen besitzt nur eine einzige Verrechnungsebene, auf der mit d'Hondt eine Stimmverrechnungsmethode der Verhältniswahl angewendet wird. Eine technische Mischung wird durch das Nebeneinander von Mehrpersonenwahlkreisen unterschiedlicher Größe sowie durch eine formale Drei-Prozent-Hürde erreicht. Die durchschnittliche Wahlkreisgröße von 6,7 täuscht auf den ersten Blick 
darüber hinweg, dass sowohl Einer-Wahlkreise vorhanden sind, in denen faktisch nach Mehrheitswahl gewählt wird, als auch sehr große Wahlkreise mit Größen bis zu 36, in denen die effektive Hürde deutlich geringer ist als in Wahlkreisen der Durchschnittsgröße und auch kleinere Parteien reelle Chancen auf einen Einzug ins Parlament besitzen.

Der Blick auf Japan, Litauen und Spanien verdeutlicht, dass sehr verschiedene technische Elemente genutzt werden können, um erstens Wahlsysteme technisch zu mischen und zweitens damit eine Balance der Repräsentationsprinzipien anzustreben. Eine eindimensionale Einordnung basierend allein auf technischen Merkmalen kann jedoch dazu führen, dass Spaniens Wahlsystem unter der technischen Verhältniswahl geführt wird (Gallagher/Mitchell 2005 a) und die Verwandtschaft bezüglich des Repräsentationsprinzips zu den Systemen Japans und Litauens leicht übersieht. Lijpharts (1994) einseitige Ausrichtung am effective threshold läuft hingegen Gefahr, substanzielle Unterschiede dieser drei Wahlsysteme zu ignorieren. Auch gemäß des typologischen Rahmens (Abbildung 1) zu einem Typ gehörende Wahlsysteme können sich also durchaus deutlich voneinander unterscheiden, wie aus Tabelle 1 ersichtlich wird. Dies zeigt die Notwendigkeit einer verfeinerten Betrachtung mit Blick auf konkrete Forschungsfragen, welche die technische Dimension in ihre Sub-Dimensionen aufteilt. Neben ihrer Rahmungsfunktion besitzt die allgemeine Typologie jedoch den zusätzlichen Nutzen, dass mit ihrer Hilfe für most similar cases design-Studien ähnliche, und zwar gemäß Abbildung 1 typologisch zu einem gemeinsamen Typ zugeordnete Fälle identifiziert und deren Effekte im Detail untersucht werden können. Gerade der Vergleich von Wahlsystemen des Typs 5 kann interessant sein, um sich systematischer als bisher der Frage zu nähern, unter welchen Umständen und basierend auf welcher Kombination technischer Elemente Mischwahlsysteme das „Beste beider Welten“ darstellen können (Lijphart 1984).

Betrachtet man die Wahlsysteme, welche dem Repräsentationsprinzip der Proportionalität folgen, lassen sich ebenfalls deutliche Unterschiede in der technischen Ausgestaltung finden. Die Niederlande und Israel sind typische Fälle für Wahlsysteme, bei denen das Repräsentationsprinzip auch technisch mit einer möglichst reinen Verhältniswahl einhergeht (allgemeiner Typ 1): Es gibt nur eine Verrechnungsebene in einem landesweiten Wahlkreis. Die Wahlkreisgröße ist somit bei gegebener Parlamentsgröße maximal. Eine gesetzliche Sperrklausel spielt entweder faktisch keine Rolle (Niederlande) ${ }^{14}$ oder befindet sich auf sehr geringem Niveau (Is-

14 Zwar besitzt das niederländische Wahlsystem eine gesetzliche Sperrklausel. Da diese jedoch dem effective threshold gleicht, hat sie keinen Einfluss auf die Wahlergebnisse (Andeweg 2005: 497). 
Eine Typologie für die vergleichende Wahlsystemforschung

rael). Gerade die Zwei-Prozent-Hürde in Israel als sehr geringe Einschränkung der Proportionalität verdeutlicht dabei jedoch, dass die Dimensionen von Wahlsystemen jeweils als kontinuierlich verstanden werden müssen und die Einordnung konkreter Fälle eben unter Berücksichtigung dieses Umstandes mithilfe weiterer Spezifikation erfolgen sollte (vgl. die Diskussion bei Pickel/Pickel 2012). Wir ordnen Israel trotz der Sperrklausel der technischen Verhältniswahl zu, betonen aber auch, dass das Vorhandensein und die Höhe der gesetzlichen Hürde dabei nicht unberücksichtigt bleiben sollten.

Mit Irland gibt es jedoch einen Fall, der auf Proportionalität abzielt und mit Single Transferable Vote auch eine entsprechende Methode benutzt, aber Wahlkreise der Größe zwischen drei und fünf besitzt. Technisch gesehen ist dieses Wahlsystem daher als gemischt einzuordnen. Carey/Hix (2011) stellen heraus, dass insbesondere Wahlsysteme mit einer Median-Wahlkreisgröße von etwa fünf bis sieben am besten dazu in der Lage sind, sowohl das Ziel der Konzentration als auch das der Proportionalität gleichzeitig zu einem zufriedenstellenden Grad zu erfüllen. Als konkrete Beispiele nennen die Autoren etwa Spanien und Irland. Während für das erste der beiden Wahlsysteme gesagt werden kann, dass es die Erreichung des „Besten beider Welten" zum Ziel hat, verdeutlicht der Fall Irland, dass das Repräsentationsprinzip bei der Beurteilung technischer Elemente und ihrer Wirkungen berücksichtigt werden muss. Während das irische Wahlsystem eindeutig mit dem Repräsentationsprinzip Proportionalität in Kraft gesetzt wurde, lässt die geringe durchschnittliche Wahlkreisgröße von 3,9 zum Beispiel Nohlen (2009 a: 368) zu der Einschätzung gelangen, dass es sich um das Repräsentationsprinzip der Mehrheitswahl handle. Hier vernachlässigt Nohlen gerade das von ihm hervorgehobene Repräsentationsprinzip des Wahlsystems und begründet seine Zuordnung zur Mehrheitswahl mit der geringen Wahlkreisgröße. So entsteht ein zumindest verkürzter Eindruck vom irischen Wahlsystem. Carey/Hix (2011: 384) bringen Irland indirekt mit der Suche nach einem „sweet spot“ auf der Dimension zwischen Proportionalität und Konzentration in Verbindung. Beide Einschätzungen übersehen Unterschiede, die sich eben konkret auf die Dimension des Repräsentationsprinzips beziehen. Der Inselstaat ist somit ein Fallbeispiel dafür, dass Repräsentationsprinzip und technische Ausgestaltung eines Wahlsystems nicht deterministisch verbunden sein müssen.

Interessant ist an dieser Stelle ebenfalls der Vergleich mit dem oben besprochenen Fall Malta. Technisch gesehen sind beide Wahlsysteme nahezu identisch; aufgrund der leicht niedrigeren durchschnittlichen Wahlkreisgröße ist Malta sogar in technischer Hinsicht marginal proportionaler. In Irland soll aber mit diesem Wahlsystem Proportionalität erreicht werden, in Malta Konzentration bzw. eine Balance beider Prinzipien. An diesen Beispielen ist somit erkennbar, dass nicht nur Wahlsysteme, 
die dem gleichen Repräsentationsprinzip folgen, sich technisch deutlich unterscheiden können, sondern dass auch umgekehrt technisch sehr ähnliche Wahlsysteme durchaus im Hinblick auf verschiedene Ziele konstruiert werden können. Rein technisch orientierte Typologien vernachlässigen solche Unterschiede nahezu vollständig.

Der deutsche Fall ist von seiner technischen Konstruktion vor allem durch das kompensatorische Element interessant. Es ist bereits schwierig, das Repräsentationsprinzip eindeutig festzustellen, was an dieser Stelle eine eindeutige Zuordnung zu einem bestimmten Typ verhindert. Die relativ hohe Fünf-Prozent-Hürde erlaubt es nicht, das Wahlsystem als technisch reines Verhältniswahlsystem im Stile der Niederlande einzuordnen. Spannend ist aber vor allem die Frage, wie die Existenz zweier Verrechnungsebenen und deren Verknüpfung zu bewerten ist. Als kompensatorisches Wahlsystem gleicht die obere Ebene Disproportionalitäten der unteren Ebene soweit wie möglich aus. Das heißt, dass im Gegensatz etwa zu Japan oder Litauen die Existenz einer Mehrheitswahlebene faktisch mitunter keine Rolle spielt. Wenngleich der Anteil der kompensierenden Mandate mit 50 Prozent relativ hoch ist, sah das für die Bundestagswahl 2009 geltende Wahlsystem die Möglichkeit vor, Überhangmandate an Parteien zu vergeben, die mehr Direktmandate gewannen als ihnen nach der Stimmenverteilung auf der oberen Ebene zustanden. Diese Regelung verhinderte oftmals eine vollständige Kompensation (z. B. 2009) und förderte so technisch zusätzlich die Konzentration des Parteiensystems (Nohlen 2009 c: 195). Interessant ist an dieser Kompensationsregelung vor allem auch, dass der Grad der Kompensation vom Wahlverhalten abhängt - so fand etwa 1976 vollständige Kompensation statt. Diese variable Konzentrationswirkung der Verbindung der Ebenen verdeutlicht erneut, dass die Dimensionen von Wahlsystemen als kontinuierlich und kontextabhängig begriffen werden müssen und technische Details zu berücksichtigen sind.

\section{Fazit}

Dem Problem, dass die vergleichende Wahlsystemforschung bis dato keine einheitliche typologische Grundlage besitzt, begegnen wir mit der Entwicklung einer allgemeinen Typologie von Wahlsystemen. Die hier vorgestellte Typologie bietet keine Mischung als weitere Alternative, sondern synthetisiert vorhandene Ansätze und liefert damit das benötigte Grundgerüst für die vergleichende Wahlsystemforschung.

Der allgemeine typologische Rahmen (Abb. 1) zieht zur Einordnung von Wahlsystemen sowohl die Dimension des Repräsentationsprinzips als auch die Dimen- 
sion der konkreten technischen Ausgestaltung eines Wahlsystems heran. Er ist hinreichend allgemein und einfach gehalten, um die an Typologien gestellte Anforderung der Komplexitätsreduktion zu erfüllen. Leitend ist dabei, dass das Repräsentationsprinzip bzw. das generelle Ziel eines Wahlsystems nicht deterministisch mit einer bestimmten technischen Ausgestaltung verbunden ist. Diese Einsicht erlaubt die Einordnung aller Wahlsysteme und trägt der zunehmenden Bedeutung von Mischwahlsystemen (hinsichtlich beider Dimensionen) Rechnung. Weiterhin wird erst über eine Berücksichtigung der generellen Zielstellung eines Wahlsystems eine sinnvolle Bewertung der Effekte möglich, indem diese eben als intendiert oder auch nicht-intendiert charakterisiert werden können. Gleichzeitig bietet die allgemeine Typologie die Möglichkeit, diese je nach Forschungsinteresse hinsichtlich der beiden Hauptdimensionen Repräsentationsprinzip und technischer Ausgestaltung zu spezifizieren.

In einem zweiten Schritt haben wir eine solche Spezifikation mit Blick auf den trade-off zwischen technischer Mehrheits- und technischer Verhältniswahl vorgenommen. Die Berücksichtigung technischer Sub-Dimensionen in einer verfeinerten Typologie (Tab. 2) unterstreicht dabei, dass die gleichzeitige, nicht-deterministische Berücksichtigung von Repräsentationsprinzip und technischer Ausgestaltung bei der Einordnung und späteren Untersuchung von Wahlsystemen unabdingbar ist. Fallbeispiele wie Irland, Malta und Spanien zeigen deutlich, dass weder ein bestimmtes Repräsentationsprinzip zu einer bestimmten technischen Ausgestaltung führen muss, noch, dass eine bestimmte technische Ausgestaltung ein klares Signal für ein bestimmtes Repräsentationsprinzip darstellt. Weiterhin wird über diese genauere Betrachtung deutlich, wie Interaktionen zwischen technischen Elementen höchst relevant für die Gesamtwirkung von Wahlsystemen sind und es demnach schwierig ist, die technische Dimension bloß über eine einzelne zusammenfassende Variable abzubilden, etwa die technische Entscheidungsregel oder die durchschnittliche Wahlkreisgröße. Trotz der Einfachheit des typologischen Rahmens lassen sich über die Sub-Dimensionen somit die relevanten Details von Wahlsystemen auch für solche Studien erfassen, die auf Feinheiten der Ausgestaltung von Wahlsystemen abheben.

Insbesondere für neue Demokratien ist die gleichzeitige Berücksichtigung von Repräsentationsprinzip und technischer Ausgestaltung zentral. Einerseits ermöglicht diese die Identifizierung erfolgreicher Designs und somit sinnvoller Vorbilder. Andererseits weist die multidimensionale Typologie nachdrücklich darauf hin, dass Repräsentationsprinzipien unter verschiedenen Rahmenbedingungen mithilfe verschiedener technischer Designs besser bzw. schlechter erreicht werden können. 
Die multidimensionale Typologie regt darüber hinaus zur Analyse bisher unberücksichtigter Forschungsfragen insbesondere mit Blick auf Mischwahlsysteme an: Welche technischen Mischungen rühren von einer Balance der Prinzipien her, welche sind mit einem polaren Repräsentationsprinzip verknüpft? Wann ist das „Beste beider Welten" gewollt, wann ist es ein Produkt anderer (sozio-politischer) Einflussfaktoren? Neben diesen inhaltlichen Fragen steht jedoch auch die methodische Herausforderung der möglichst exakten Feststellung des Repräsentationsprinzips. Unsere Analyse hat einen ersten Ansatzpunkt geliefert. Weiterhin ist es aber wichtig, zu versuchen, Repräsentationsprinzipien möglichst exakt festzustellen und nach Möglichkeit über eine kategoriale Einschätzung (wie wir sie vorgenommen haben) hinauszugehen. So ließe sich ebenfalls ein möglicher Wandel des Repräsentationsprinzips pointierter feststellen. Dies könnte unter anderem für die Analyse der deutschen Reformdebatte zentral sein.

An dieser Stelle sei nochmals darauf hingewiesen, wie fruchtbar und notwendig eine enge Zusammenarbeit zwischen eher qualitativ ausgerichteten Forschern, die sich intensiv mit den Entstehungshintergründen von Wahlsystemen und deren intendierten Zielen auseinandersetzen, und quantitativ ausgerichteten Vertretern ist, die die Wirkungen bestimmter technischer Elemente und deren Kombinationen vergleichend analysieren. Die langfristigen Ziele sind dann eine komplette empirische Einordnung von Wahlsystemen und die systematische Untersuchung der Auswirkungen von Kombinationen unterschiedlicher technischer Elemente. Nur auf dieser Grundlage können Wahlsystemdesigner - ausgerichtet an konkreten Repräsentationsprinzipien - sinnvoll Vorschläge für technische Regeln unterbreiten, wenn Wahlsysteme zu entwickeln oder zu reformieren sind. Die bisherige einseitige Ausrichtung entweder auf die technische Ausgestaltung oder auf die Repräsentationsprinzipien vermag dies nicht zu leisten.

\section{Literatur}

Andeweg, Rudy B., 2005: The Netherlands: the sanctity of proportionality, in: Michael Gallagher/Paul Mitchell (Hrsg.), The Politics of Electoral Systems, Oxford, 491-510.

Behnke, Joachim, 2011: Grundsätzliches zur Wahlreformdebatte, in: Aus Politik und Zeitgeschichte 4/2011, 14-21.

Benoit, Kenneth, 2002: The endogeneity problem in electoral studies: a critical reexamination of Duverger's mechanical effect, in: Electoral Studies 21, 35-46. 
Eine Typologie für die vergleichende Wahlsystemforschung

Benoit, Kenneth, 2007: Electoral laws as political consequences: explaining the origins and change of electoral institutions, in: Annual Review of Political Science 10 (1), 363-390.

Blais, André/Massicotte, Louis, 1997: Electoral formulas: a macroscopic perspective, in: European Journal of Political Research 32, 107-129.

Bowler, Shaun/Farrell, David M./Pettitt, Robin T., 2005: Expert opinion on electoral systems: so which electoral system is "best"?, in: Journal of Elections, Public Opinion \& Parties 15 (1), 3-19.

Bundesverfassungsgericht (BVerfG), 1997: Zur Verfassungsmäßigkeit von Überhangmandaten, die ohne Verrechnung anfallen oder ohne Ausgleichsmandate zugeteilt werden. Urteil (2 BvF 1/95) des Bundesverfassungsgerichts, o. O.

Carey, John M./Hix, Simon, 2011: The electoral sweet spot: low-magnitude proportional electoral systems, in: American Journal of Political Science 55 (2), 383-397.

Collier, David/Laporte, Jody/Seawright, Jason, 2008: Typologies: forming concepts and creating categorial variables, in: Janet M. Box-Steffensmeier/Henry E. Brady/David Collier (Hrsg.), The Oxford Handbook of Political Methodology, Oxford/New York, 152-173.

Collier, David/Laporte, Jody/Seawright, Jason, 2012: Putting typologies to work: concept formation, measurement, and analytic rigor, in: Political Research Quarterly 65 (1), 217-232.

Cox, Gary W., 1997: Making Votes Count. Strategic Coordination in the World's Electoral Systems, Cambridge.

Decker, Frank, 2011: Brauchen wir ein neues Wahlrecht?, in: Aus Politik und Zeitgeschichte 4/2011, 3-9.

Dunleavy, Patrick/Margetts, Helen, 1995: Understanding the dynamics of electoral reform, in: International Political Science Review 16 (1), 9-29.

Duverger, Maurice, 1984: Which is the best electoral system?, in: Arend Lijphart/ Bernard Grofman (Hrsg.), Choosing an Electoral System. Issues and Alternatives, Westport, 31-39.

Farrell, David M., 2011: Electoral Systems. A Comparative Introduction, New York (2. Aufl.).

Ferrara, Federico/Herron, Erik S./Nishikawa, Misa, 2005: Mixed Electoral Systems. Contamination and its Consequences, New York.

Gallagher, Michael, 2005: Ireland: the discrete charm of PR-STV, in: Michael Gallagher/Paul Mitchell (Hrsg.), The Politics of Electoral Systems, Oxford, 511-532. 
Gallagher, Michael/Mitchell, Paul (Hrsg.), 2005 a: The Politics of Electoral Systems, Oxford.

Gallagher, Michael/Mitchell, Paul, 2005 b: Introduction to electoral systems, in: Michael Gallagher/Paul Mitchell (Hrsg.), The Politics of Electoral Systems, Oxford, 3-23.

Gerring, John, 2012: Mere description, in: British Journal of Political Science 42 (4), 721-746.

Gschwend, Thomas, 2007: Ticket-splitting and strategic voting under mixed electoral rules. Evidence from Germany, in: European Journal of Political Research 46, 1-23.

Hirczy, Wolfgang, 1995: Explaining near-universal turnout: the case of Malta, in: European Journal of Political Research 27, 255-272.

Hopkin, Jonathan, 2005: Spain: proportional representation with majoritarian outcomes, in: Michael Gallagher/Paul Mitchell (Hrsg.), The Politics of Electoral Systems, Oxford, 375-394.

Jacobs, Kristof/Leyenaar, Monique, 2011: A conceptual framework for major, minor, and technical electoral reform, in: West European Politics 34 (3), 495-513.

Jenkins Commission, 1998: Voting Systems: The Jenkins Report. Research Paper 98/112, House of Commons Library, London.

Jesse, Eckhard, 2009: Verhältniswahl und Gerechtigkeit, in: Zeitschrift für Politikwissenschaft, Sonderheft 19 „Wahlsystemreform“, 105-131.

Kaiser, André, 2002: Gemischte Wahlsysteme. Ein Vorschlag zur typologischen Einordnung, in: Zeitschrift für Politikwissenschaft 12 (4), 1545-1571.

LeDuc, Lawrence, 2011: Electoral reforms and direct democracy in Canada: when citizens become involved, in: West European Politics 34 (3), 551-567.

Lijphart, Arend, 1984: Trying to have the best of both worlds: semi-proportional and mixed systems, in: Arend Lijphart/Bernard Grofman (Hrsg.), Choosing an Electoral System. Issues and Alternatives, New York, 207-213.

Lijphart, Arend, 1994: Electoral Systems and Party Systems. A Study of TwentySeven Democracies, 1945-1990, Oxford/New York.

Lijphart, Arend/Grofman, Bernard (Hrsg.), 1984 a: Choosing an Electoral System. Issues and Alternatives, New York.

Lijphart, Arend/Grofman, Bernard, 1984 b: Choosing an electoral system, in: Arend Lijphart/Bernard Grofman (Hrsg.), Choosing an Electoral System. Issues and Alternatives, New York, 3-12.

Linhart, Eric, 2009: Mögliche Auswirkungen von Grabenwahlsystemen in der Bundesrepublik Deutschland. Theoretische Überlegungen und Simulationen, in: Zeitschrift für Parlamentsfragen 40 (3), 637-660. 
Eine Typologie für die vergleichende Wahlsystemforschung

Lipset, Seymour M./Rokkan, Stein (Hrsg.), 1967: Party Systems and Voter Alignments: Cross-National Perspectives, New York.

Massicotte, Louis, 2005: Canada: sticking to First-Part-the-Post, for the time being, in: Michael Gallagher/Paul Mitchell (Hrsg.), The Politics of Electoral Systems, Oxford, 99-118.

Massicotte, Louis/Blais, André, 1999: Mixed electoral systems: a conceptual and empirical survey, in: Electoral Studies 18, 341-366.

Nohlen, Dieter, 1978: Wahlsysteme der Welt: Daten und Analysen, München.

Nohlen, Dieter, 1984: Two incompatible principles of representation, in: Arend

Lijphart/Bernard Grofman (Hrsg.), Choosing an Electoral System. Issues and Alternatives, New York, 83-89.

Nohlen, Dieter, 2009 a: Wahlrecht und Parteiensystem. Zur Theorie und Empirie der Wahlsysteme, Opladen (6. Aufl.).

Nohlen, Dieter, 2009 b: Wahlsysteme in Reformprozessen, in: Zeitschrift für Politikwissenschaft, Sonderheft 19 „Wahlsystemreform“, 45-80.

Nohlen, Dieter, 2009 c: Erfolgswertgleichheit als fixe Idee oder: Zurück zu Weimar? Zum Urteil des Bundesverfassungsgerichts über das Bundeswahlgesetz vom 3. Juli 2008, in: Zeitschrift für Parlamentsfragen 40 (1), 179-195.

Norris, Pippa, 2000: Women: representation and electoral systems, in: Richard Rose (Hrsg.), International Encyclopedia of Elections, London, 348-351.

Pappi, Franz Urban/Herrmann, Michael, 2010: Überhangmandate ohne negatives

Stimmgewicht: Machbarkeit, Wirkungen, Beurteilung, in: Zeitschrift für Parlamentsfragen 41 (2), 260-278.

Pickel, Susanne/Pickel, Gert, 2012: Die Messung von Indizes in der Vergleichenden Politikwissenschaft - methodologische Spitzfindigkeit oder substantielle Notwendigkeit, in: Zeitschrift für Vergleichende Politikwissenschaft 6 (1, Supplement), 1-17.

Prittwitz, Volker von, 2011: Hat Deutschland ein demokratisches Wahlsystem?, in:

Aus Politik und Zeitgeschichte 4/2011, 9-14.

Proctor, J.H., 1980: The acceptance of Proportional Representation in Malta, in:

Parliamentary Affairs 33 (1), 308-321.

Rae, Douglas W., 1967: The Political Consequences of Electoral Laws, New Haven.

Rahat, Gideon/Hazan, Reuven Y., 2005: Israel: the politics of an extreme electoral system, in: Michael Gallagher/Paul Mitchell (Hrsg.), The Politics of Electoral Systems, Oxford, 333-351.

Reed, Steven R., 2005: Japan: haltingly towards a two-party system, in: Michael Gallagher/Paul Mitchell (Hrsg.), The Politics of Electoral Systems, Oxford, 277-293. 
Rose, Richard, 1984: Electoral systems: a question of degree or principle?, in: Arend Lijphart/Bernard Grofman (Hrsg.), Choosing an Electoral System. Issues and Alternatives, New York, 73-81.

Saalfeld, Thomas, 2005: Germany: stability and strategy in a mixed-member proportional system, in: Michael Gallagher/Paul Mitchell (Hrsg.), The Politics of Electoral Systems, Oxford, 209-229.

Sakamoto, Takayuki, 1999: Explaining electoral reform. Japan versus Italy and New Zealand, Party Politics 5 (4), 419-438.

Sartori, Giovanni, 1970: Concept misformation in comparative politics, in: The American Political Science Review 64 (4), 1033-1053.

Sartori, Giovanni, 1997: Comparative Constitutional Engineering. An Inquiry into Structures, Incentives, and Outcomes, New York (2. Aufl.).

Schmitt-Beck, Rüdiger, 2012: Empirische Wahlforschung in Deutschland: Stand und Perspektiven zu Beginn des 21. Jahrhunderts, in: Politische Vierteljahresschrift 45, Sonderheft „Wählen in Deutschland“, 2-38.

Schoen, Harald, 2005: Wahlsystemforschung, in: Jürgen W. Falter/Harald Schoen (Hrsg.), Handbuch Wahlforschung, Wiesbaden, 573-607.

Schoen, Harald, 2007: Eine optimale Lösung? Eine Replik auf Gerd Strohmeier in Heft 3/2007 der Zeitschrift für Parlamentsfragen, in: Zeitschrift für Parlamentsfragen 38 (4), 862-865.

Shugart, Matthew S., 2005: Comparative electoral systems research: the maturation of a field and new challenges ahead, in: Michael Gallagher/Paul Mitchell (Hrsg.), The Politics of Electoral Systems, Oxford, 25-55.

Shugart, Matthew S./Wattenberg, Martin P. (Hrsg.), 2001 a: Mixed-Member Electoral Systems. The Best of Both Worlds?, Oxford.

Shugart, Matthew S./Wattenberg, Martin P., 2001 b: Mixed-member electoral systems: a definition and typology, in: Matthew S. Shugart/Martin P. Wattenberg (Hrsg.), Mixed-Member Electoral Systems. The Best of Both Worlds?, Oxford, 9-24.

Strohmeier, Gerd, 2009: Vergangene und zukünftige Reformen des deutschen Wahlsystems, in: Zeitschrift für Politikwissenschaft, Sonderheft 19 „Wahlsystemreform", 11-44.

Supreme Court, 1991: Reference re Prov. Electoral Boundaries (Sask.). Judgment ([1991] 2 S.C.R. 158) of the Supreme Court of Canada, o. O. 
Korrespondenzanschrift:

Johannes Raabe, M.Sc.

Universität Kiel

Institut für Agrarökonomie

Wilhelm-Seelig-Platz 7

24098 Kiel

E-Mail: jraabe@ae.uni-kiel.de

Prof. Dr. Eric Linhart

Universität Kiel

Institut für Agrarökonomie

Wilhelm-Seelig-Platz 7

24098 Kiel

E-Mail: eric.linhart@ae.uni-kiel.de 